\title{
Sound Level Meter as Software Application
}

\begin{abstract}
R. BARAŃSKI*
AGH University of Science and Technology, Faculty of Mechanical Engineering and Robotics, Department of

Mechanics and Vibroacoustics

Al. A. Mickiewicza 30, 30-059 Krakow, Poland

Nowadays, technical possibilities to build and implement new solution in industry or scientists research are very wide. PC computer with dedicated environmental software makes building equipment previously reserved to FPGA systems possible. In this article we focus on building a sound level meter application. The main reason to undertake this problem was to check precision of sound level meter consisting of a dedicated computer program and the main board integrated sound card. All algorithms were implemented in LabVIEW 8.6, thus we call our system SLM LV v1.2c. In testing SLM LV v1.2c we have performed the calibration procedure in the Vibroacoustics Laboratory of the Department of Mechanics and Vibroacoustics (a Calibration Laboratory accredited by Polish Centre for Accreditation). The presented results show that our meter in a specific range meets requirements for 1st class sound level meters. Certainly, we can not use it as regular sound level meter without any other research like e.g. type approval, but at the same time we can consider it to be an inexpensive sound level indicator.At the end of the paper, we present results of comparison between measurements obtained with SLM_LV_v1.2c and the popular sound level meter SVAN 945A and show some sources of errors.
\end{abstract}

DOI: 10.12693/APhysPolA.125.A-66

PACS: 43.38.Kb; 43.58.Dj

\section{Introduction}

Professional sound level meters are expensive. Thus, there is a necessity to make some equivalent of the professional sound level meter [1]. In many measurements we do not need high precision results. Sometimes few decibels of measuring accuracy will be enough.

To quote point 5.1.1 PN-EN 61672-1 “... Sound level meter consists of microphone, signal processor and indicate system..." [2]. In our research we attempted to build a sound level meter we use standard components like laptop Dell E1405 with integrated sound card (16 bit SigmaTel High Definition Audio CODEC). Tests were also made with a low-cost multifunction DAQ card (14bit $48 \mathrm{~kb} / \mathrm{s}$ National Instruments).

The last element - the microphone - was the most disputable. Low cost microphones have nonstandard dimensions. For some tests it was a significant obstacle because calibration and measuring equipment needs to meet certain standards. In this tests we used GRAS 40AF with preamplifier GRAS type 12AA.

Software was implemented in LabView 8.6 with Sound and Vibration Analysis Toolkit version 6.0. This is a popular environmental for signal measurement and analysis [3-5].

\section{Research}

A sound level meter must fulfil requirements of PN-EN 61672-1. Our proposal does not fully meet this norm because the goal was to build a device for not high accuracy

*e-mail: robertb@agh.edu.pl measurements with the assumption that results close to professional ones will be enough. However, to check its accuracy, we have performed the same tests that are applicable periodically to professional sound level meters, namely the calibration tests $[1,6,7]$.

The presented research was carried out in the accredited calibration Vibroacoustics Laboratory which uses procedure based on PN-EN 61672-3 [7]. According to this procedure, we needed to use a $1 \frac{1}{2}$ or $\frac{1}{4}$ inch microphone and an electrostatic actuator. That was the main reason to use very precise microphone GRAS $40 \mathrm{AF}$ with preamplifier GRAS type 12AA.

\subsection{Calibration procedure}

During the tests, environmental condition were in accordance with PN-EN 61672-3 [7]. Below we present the most important result of our investigation. The following abbreviations have been used in the tables below: Pec 1,2 - Permissible error class 1,2 [dB], A, C, LIN - frequency weight characteristic, Fast (F), Slow (S) - timeweight characteristic, SPL - sound pressure level [dB], LEQ - equivalent level [dB], $F_{\max }$ - maximum value for time weighting F [dB], $S_{\max }$ - maximum value for time weighting $\mathrm{S}[\mathrm{dB}], L$ - level for steady signal $[\mathrm{dB}]$, $L_{i}$ - tonebursts level [dB], $L_{\mathrm{C}}$ - level for steady signal with frequency weights $\mathrm{C}, \delta_{\text {ref }}-$ reference value $[\mathrm{dB}]$, $L_{p}$ - positive one-half-cycle level [dB], $L_{n}$ - negative one-half-cycle level [dB],

\subsubsection{Frequency and time weighting at $1 \mathrm{kHz}$}

Performed by steady sinusoidal electrical input signal used for A, C, LIN frequency weighting and $F, S$ time-weighting sound level. All values in Error row are computed as $L_{\ldots} \ldots-L_{\mathrm{A}, \mathrm{F}}$ (e.g. for 'C' column, value is $\left.L_{\mathrm{C}}-L_{\mathrm{A}, \mathrm{F}}\right)$. Results are presented in Table I. 
The examined system SLM_LV_v1.2c met this requirement for a 1st class sound level meter.

\subsubsection{Acoustical signal tests with frequency weighting}

In this part we used electrostatic actuator and SVAN
403 generator. The examined system SLM_LV_v1.2c met the requirement for a 1st class sound level meter when it refers to differences for frequency weighting A and $\mathrm{C}$.

Frequency and time weightings at $1 \mathrm{kHz}$.

TABLE I

\begin{tabular}{|c|c|c|c|c|c|}
\hline Characteristic & \multicolumn{3}{|c|}{ A } & $\mathrm{C}$ & LIN \\
\hline Time-weighted & Fast & Slow & - & Fast & Fast \\
\hline Result & SPL & SPL & LEQ & SPL & SPL \\
\hline Level [dB] & $93.98\left(L_{\mathrm{A}, \mathrm{F}}\right)$ & $93.98\left(L_{\mathrm{A}, \mathrm{S}}\right)$ & $93.98\left(L_{\mathrm{A}, \mathrm{LEQ}}\right)$ & $93.99\left(L_{\mathrm{C}}\right)$ & $94.00\left(L_{\mathrm{Z}(\mathrm{LIN})}\right)$ \\
\hline Error $\left(L \ldots-L_{\mathrm{A}, \mathrm{F}}\right)[\mathrm{dB}]$ & - & 0 & 0 & 0.01 & 0.02 \\
\hline Pec $1,2[\mathrm{~dB}]$ & \multicolumn{3}{|c|}{ \pm 0.3} & \pm 0.4 & \pm 0.4 \\
\hline
\end{tabular}

Toneburst response.

TABLE II

\begin{tabular}{c|c|c|c|c|c|c|c}
\hline \hline Duration time [ms] & Time-weighted & $L$ & $L_{i}$ & $L_{i}-L$ & $\delta_{\text {ref }}$ & $\left(\left(L_{i}-L\right)-\delta_{\text {ref }}\right)$ & Pec 1,2 \\
\hline \multirow{3}{*}{200} & $F_{\max }$ & 117.0 & 117.0 & 0.0 & -1.0 & $\mathbf{1 . 0}$ & \pm 0.8 \\
& $S_{\max }$ & 117.0 & 110.4 & -6.6 & -7.4 & $\mathbf{0 . 8}$ & \pm \\
& $L_{\mathrm{AE}}$ & 117.0 & 110.0 & -7.0 & -7.0 & $\mathbf{0 . 0}$ & \\
\hline \multirow{3}{*}{2} & $F_{\max }$ & 117.0 & 99.4 & -17.6 & -18.0 & $\mathbf{0 . 4}$ & $+1.3 ;-1.8$ \\
& $S_{\max }$ & 117.0 & 90.4 & -26.6 & -27.0 & $\mathbf{0 . 4}$ & $+1.3 ;-3.3$ \\
& $L_{\mathrm{AE}}$ & 117.0 & 90.0 & -27.0 & -27.0 & $\mathbf{0 . 0}$ & $+1.3 ;-1.8$ \\
\hline \multirow{2}{*}{0.25} & $F_{\max }$ & 117.0 & 90.2 & -26.8 & -27.0 & $\mathbf{0 . 2}$ & $+1.3 ;-3.3$
\end{tabular}

\subsubsection{Self-generated noise}

Microphone impedance was replaced by the substitute impedance. For three different frequency-weights sound level result were as follows: $43.09 \mathrm{~dB}(\mathrm{~A}), 46.11 \mathrm{~dB}(\mathrm{C})$, and $68.36 \mathrm{~dB}(\mathrm{LIN})$

\subsubsection{Level linearity in the reference level range}

Test based on steady sinusoidal electrical signal at a frequency of $8 \mathrm{kHz}$. The tested device has been set to $\mathrm{A}$ (frequency weighting) and Fast (integration time).

The examined system SLM_LV_v1.2c met the linearity requirement for a 1 st class sound level meter within the range 120-65 dB, (see Fig. 1).

\subsubsection{Toneburst response}

This test checks response of a sound level meter to short-duration signals with $4 \mathrm{kHz}$ tonebursts that start and stop at zero crossing and are extracted from a steady $4 \mathrm{kHz}$ sinusoidal electrical input signal. The tested device has been set to A (frequency weighting) and Fast/Slow (integration time). The adopted toneburst times were $200 \mathrm{~ms}, 2 \mathrm{~ms}$, and $0.25 \mathrm{~ms}$. Results are presented in Table II.

The examined system SLM_LV_v1.2c met the requirement for a 1st class sound level meter except for the toneburst duration time $200 \mathrm{~ms}$ and Fast integration time.

\subsubsection{Peak $C$ sound level}

The testing signals were a single sinusoidal complete cycle of $8 \mathrm{kHz}$ and a positive and negative half sinusoidal cycle of $500 \mathrm{~Hz}$. All signals were started and stopped at zero crossing. Peak $\mathrm{C}$ sound level measurement uncertainty equals $0.2 \mathrm{~dB}$. Results are presented in Table III.

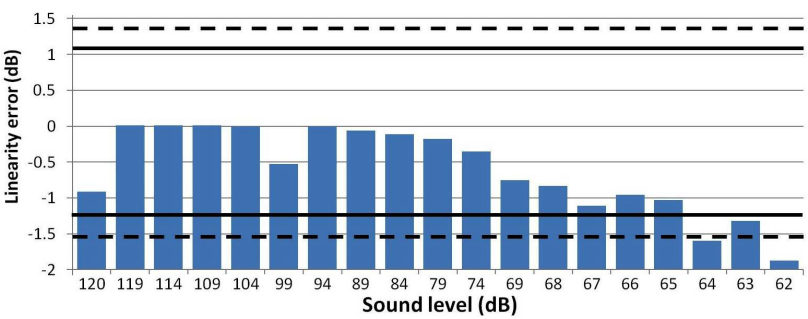

Fig. 1. Linearity level (solid line: area of 1st class permission error; dotted line: area of 2 nd class permission error).

The examined system SLM_LV_v1.2c met the requirement in question for a 1st class sound level meter.

\subsubsection{Overload indication}

This part of the calibration test checks differences between the levels of the positive and negative $4 \mathrm{kHz}$ onehalf-cycle input signal that first caused the displays of overload indication. Results are presented in Table IV.

The examined system SLM_LV_v1.2c met this requirement for a 1st class sound level meter.

\subsection{Comparison to SVAN $945 A$}

In order to show all capabilities of SLM LV v1.2c system, there has been comparative study carried out to adhere to the 1st class sound level meter SVAN 945A. Measurements have been carried out in anechoic chamber to reduce uncontrolled distortions. During parallel measurements we have compared sound pressure levels. 
Peak C sound level.

\begin{tabular}{l|c|c|c|c|c|c|c}
\hline \hline Signal cycle & Frequency $[\mathrm{Hz}]$ & $L_{\mathrm{C}}$ & $L_{\text {Cpeak }}$ & $L_{\text {Cpeak }}-L_{\mathrm{C}}$ & $\delta_{\text {ref }}[\mathrm{dB}]$ & $\left(\left(L_{\text {Cpeak }}-L_{\mathrm{C}}\right)-\delta_{\text {ref }}\right)$ & Pec 1,2 \\
\hline One & 8000 & 112 & 115 & 3.0 & 3.4 & $-\mathbf{0 . 4}$ & \pm 2.4 \\
\hline Positive half & 500 & 112 & 114.2 & 2.2 & 2.4 & $-\mathbf{0 . 2}$ & \pm 1.4 \\
\hline Negative half & 500 & 112 & 114.2 & 2.2 & 2.4 & $\mathbf{- 0 . 2}$ & \pm 1.4
\end{tabular}

Overload indication.

TABLE IV

\begin{tabular}{|c|c|c|c|c|}
\hline \multirow{2}{*}{$\begin{array}{c}L \\
{[\mathrm{~dB}]}\end{array}$} & \multicolumn{2}{|c|}{ Overload indication } & \multirow{2}{*}{$\begin{array}{c}\left|L_{\mathrm{n}}-L_{\mathrm{p}}\right| \\
{[\mathrm{dB}]}\end{array}$} & \multirow{2}{*}{$\begin{array}{c}\text { Pec } 1,2 \\
{[\mathrm{~dB}]}\end{array}$} \\
\hline & $L_{\mathrm{n}}[\mathrm{dB}]$ & $L_{\mathrm{p}}[\mathrm{dB}]$ & & \\
\hline 119 & 81.8 & 81.9 & 0.1 & 1.8 \\
\hline \multicolumn{3}{|c|}{ Overload indication block } & \multicolumn{2}{|c|}{ YES } \\
\hline
\end{tabular}

As a source we have used pink noise and omnidirectional sound source. During the measurement session there were only microphones and the sound source placed in the anechoic chamber. PC with software SLM LV v1.2c and SVAN945A analyser have been placed outside the chamber. Fig. 2 shows positions of the meters and the sound source. For both devices, the same parameters have been used (time $30 \mathrm{~s}$, linear correction filter, Fast characteristics).

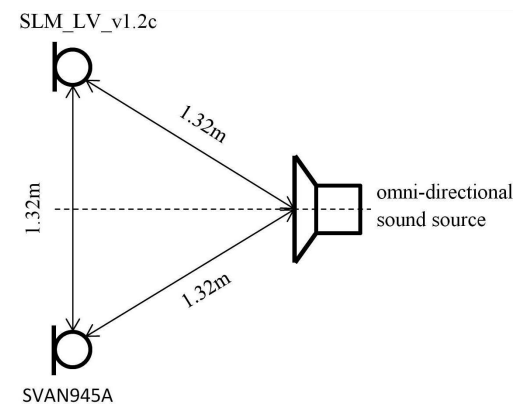

Fig. 2. Schematic diagram of the measurement setup.

Initial measurements have been carried out with the calibration of both devices (Norsonic 1251). SLM_LV_v1.2c equipped with non-professional (low cost) microphone Panasonic WM-61A did not allow correct calibration as its diameter did not match the diameter of the calibrator hole $(1 / 2$ inch). Due to this fact, the sound pressure level has changed. It had significant impact on the difference between levels measured by both devices. Measurement results are presented in Figure 3a. Difference in total equivalent level $\left(L_{\text {eq }}\right)$ between SVAN 945A and SLM_LV_v1.2c exceeded $12.0 \mathrm{~dB}$.

Better results have been provided with the calibration based on measurement with SVAN 945A, performed for a control signal (white noise at $80 \mathrm{~dB}$ ). There has been a correction $(K)$ determined for SLM_LV_v1.2c based on the difference between equivalent levels measured by SVAN $945 \mathrm{~A}\left(L_{\mathrm{SVAN}}\right)$ and the SLM_LV_v1.2c $\left(L_{\mathrm{SLM}}\right.$ LV $)$ :

$$
K=L_{\text {SVAN }}-L_{\text {SLM_LV }} \text {. }
$$

Figure $3 \mathrm{~b}$ presents an example of the results obtained from measurements taken after the calibration based on
SVAN 945A. In this case, the difference between summary level of $L_{\text {eq }}$ for SVAN 945A and SLM_LV_v1.2c has not exceeded $-2 \mathrm{~dB}$. For particular bands between $20 \mathrm{~Hz}$ and $16 \mathrm{kHz}$, the average difference was fluctuating on the $-1 \mathrm{~dB}$ level (SLM LV v1.2c used to underestimate results in comparison to $\overline{\mathrm{S} V A N} 945 \mathrm{~A}$ ).

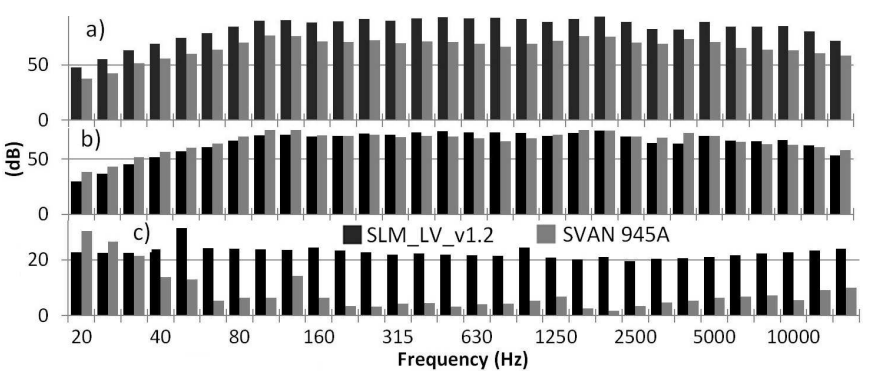

Fig. 3. Measurement results: (a) calibration based on Norsonic 1251; (b) calibration based on SVAN 945A; (c) background measurements.

Measurements of the background sound level in the chamber have also been made. According to the results presented in 2.1.4, there were significant errors obtained for lower levels. In terms of the anechoic chamber's background level SVAN 945A, provided a difference of $46 \mathrm{~dB}$. An example of spectrum for background measurements is presented in Figure 3c.

\subsection{Sources of errors}

Apart from software, SLM L LV_v1.2c sound meter is based on PC sound card and a microphone. Comparative measurements have been performed with some other integrated sound cards (dedicated to PC models such as: IBM, Dell, Acer, Lenovo, Toshiba, California Access). All sound cards input have been exposed to the same pink noise electric signal . All computers have used Windows operating system. Pre-gain has been set to $0 \mathrm{~dB}$ and the microphone level to the maximum.

Figure 4a presents measurement results for the signal after $1 / 3$ octave filters have been used. The solid line stands for devices equipped with High Definition Audio (HD Audio) sound card that meets specification for the audio sub-system of Personal Computers released by Intel in 2004 [9]. The dotted line stands for older laptops 
equipped with sound cards other than HD Audio. The frequency response of converters of HD Audio cards differs in the pregain level and keeps characteristic flat up to $10 \mathrm{kHz}$.

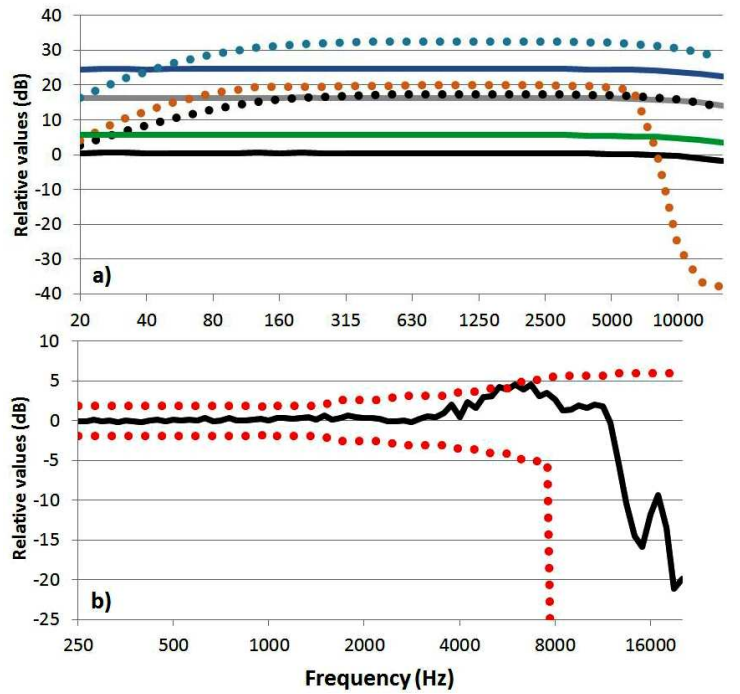

Fig. 4. Frequency characteristics: (a) sound card (solid line: devices equipped with HD Audio; dotted line: laptops equipped with sound cards other than HD Audio), (b) microphone (solid line: measurement results; dotted line: tolerance limits for class 2 sound level meters) [8].

We also noticed that power supply or battery had no impact on the measurements results.

Figure $4 \mathrm{~b}$ represents frequency characteristics of the inexpensive microphone Digitus (the solid line) [8]. Measurements were made in anechoic chamber in free field conditions, for wave incidence angle $0^{\circ}$. Dotted lines represent tolerance limits for frequency characteristics of class 2 sound level meters, conformant to the requirements of Part 1 of the standard PN-EN 61672 [10].

On the basis of the figures mentioned above it can be said that the main factor responsible for nonlinearity of the characteristic of the system is the microphone, causing significant distortions.

\section{Conclusion}

In this article we presented the result of our research. We have performed a full calibration test based on PNEN 61672-3:2007 standard using software sound level meter based on low-cost elements (standard laptop with integrated sound card) and comparative studies with professional meter SVAN 945A.

Low-cost microphones are not subject to standardisation of geometric or electric parameters. This requirement is especially important in:

- System calibration. Calibrator standards are $1 \frac{1}{2}$ or $\frac{1}{4}$ inch. In particular situation, we can make our own module to fit our microphone to calibrator.
- Self-generated noise. In the laboratory, a substitution impedance was used. This property is provided by producer. Very often, low-cost microphones do not have any specifications or data are not precise enough.

- Acoustical signal tests of frequency weighting. In this case, electrostatic actuator was used. This is the only one way to make this test. In the case of a non-standard microphone, the best method will be a free-field facility. Laboratory used only electrostatic actuator.

The other parts of calibration test were made without microphone by using electrical input signal.

SLM LV v1.2c sound level meter passed almost all tests to be classified as a 1st class sound level meter. Only for a part of one test (Toneburst response) SLM_LV_v1.2c failed the exam. Using a different card (low-cost NI USB 6009) allowed our system to pass it.

The results of the comparative study with the 1st class sound level meter SVAN 945A showed that in case of proper calibration of the SLM_LV_v1.2c, values obtained for entire acoustic band $\overline{\text { differ }}$ from professional meter's results by no more than $2 \mathrm{~dB}$.

However, it needs to be said that the level of distortion for SLM_LV_v1.2c is significantly higher. Real sound level to which it can be applied is around $60 \mathrm{~dB}$.

Where the measurement system is built with sound cards compatible with High Definition Audio standard, the main source of nonlinearity of frequency characteristic is the microphone.

On the basis of the presented research we can say that the system built out of low-cost elements performed the majority of standard periodic tests applicable to sound level meters. What is very important is that the system (software and hardware) was tested only in few standard tests of all and we must realise that this is not a professional sound level meter. It can be used in situations when we do not expect high accuracy. Without additional tests we can not interpret result as the same as those obtained with professional sound level meters. This distinction means that the appropriate name for this type of apparatus would be the sound level indicator rather than the sound level meter.

\section{Acknowledgments}

The study described in this paper has been performed within the project No. 11.11.130.885.

\section{References}

[1] G. Wszołek, Acta Phys. Pol. A 119, 1081 (2011).

[2] PN-EN 61672-1:2005 Electroacoustics - Sound level meters, Part 1: Specifications, PKN, Warszawa 2005.

[3] M. Jabłoński, A. Ozga, T. Korbiel, P. Pawlik, Acta Phys. Pol. A 119, 977 (2011) 
[4] R. Barański, W. Batko, Acta Phys. Pol. A 119, 913 (2011).

[5] R. Trojanowski. J. Wiciak, Acta Phys. Pol. A 118, 168 (2010).

[6] Zb. Engel, G. Wszołek, Archiv. Acoust. 29, 283 (2004).

[7] PN-EN 61672-3:2007 Electroacoustics - Sound level meters, Part 3: Periodic tests, PKN, Warszawa 2007.

[8] R. Barański, G. Wszołek, Archiv. Acoust. 38, 19 (2013).
[9] Intel ${ }^{\circledR}$ High Definition Audio 1.0a Specification, Intel Corporation, 2013.

[10] PN-EN 61672 Electroacoustics - Sound level meters; Part 1: Specifications , Part 2: Pattern evaluation tests (2005), Part 3: Periodic tests, PKN, Warszawa $2000,2005$. 